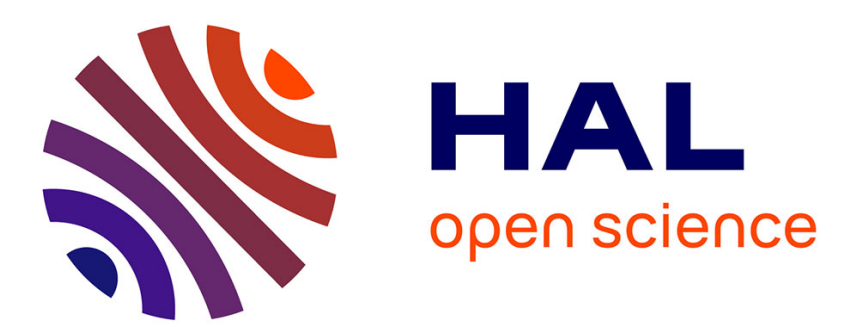

\title{
Particle trajectories and size sorting above a rippled bed under standing water waves
}

\author{
T. Chu, A. Jarno-Druaux, François Marin, A. Ezersky
}

\section{To cite this version:}

T. Chu, A. Jarno-Druaux, François Marin, A. Ezersky. Particle trajectories and size sorting above a rippled bed under standing water waves. Physical Review E : Statistical, Nonlinear, and Soft Matter Physics, 2012, 85 (2), 10.1103/PhysRevE.85.021304 . hal-02074558

\section{HAL Id: hal-02074558 https://hal.science/hal-02074558}

Submitted on 20 Mar 2019

HAL is a multi-disciplinary open access archive for the deposit and dissemination of scientific research documents, whether they are published or not. The documents may come from teaching and research institutions in France or abroad, or from public or private research centers.
L'archive ouverte pluridisciplinaire HAL, est destinée au dépôt et à la diffusion de documents scientifiques de niveau recherche, publiés ou non, émanant des établissements d'enseignement et de recherche français ou étrangers, des laboratoires publics ou privés. 


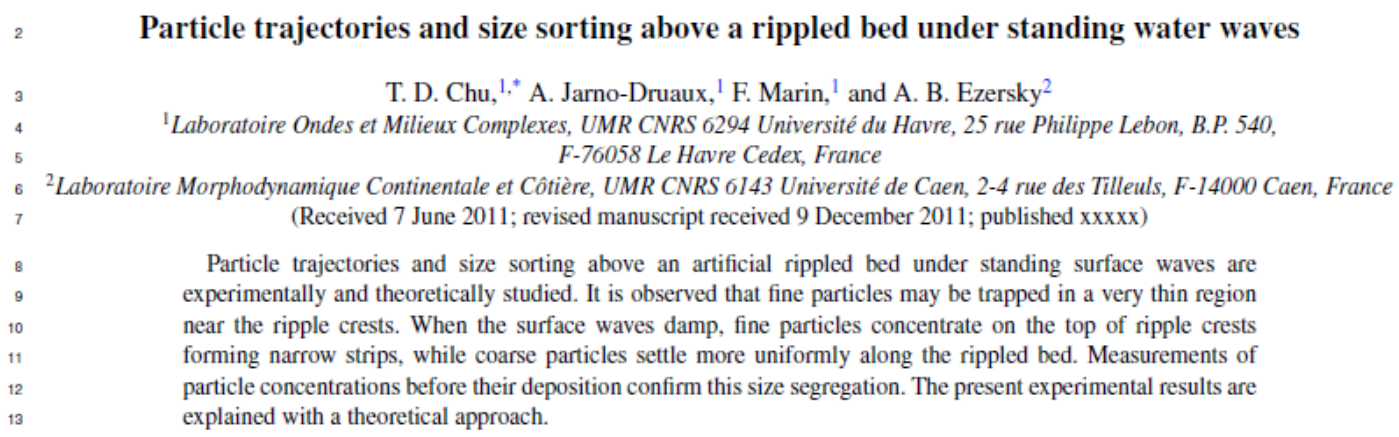

\section{INTRODUCTION}

Periodic patterns with typical wavelengths of $\approx 10 \mathrm{~cm}$ often 17 cover seabeds in coastal zones. These structures, generally 18 called ripples, result from complex interactions between the 19 flow of water and the granular bottom. Since the pioneering 20 works of Ayrton [1] and Bagnold [2], the formation of vortex 21 ripples induced by oscillatory motion has been intensively 22 studied. Many experimental and theoretical works have been 23 conducted on the morphology of these structures, which 24 significantly affect sediment transport, wave damping, and 25 boundary layer structure. The wavelength of ripples is roughly 26 proportional to the amplitude of the water motion [3], and 27 the flow in their vicinity is dominated by the generation 28 and ejection of lee-side coherent vortices [4-6]. Detailed 29 visualizations of vortex dynamics have been carried out [7-9].

A majority of the literature devoted to ripple patterns 31 considers the case of homogeneously sized grains [10-12]; 32 however, sediments in coastal zones are usually heterogeneous ${ }_{33}$ in size. It should be noted that pattern formation in granular 34 materials containing different particles is widely investigated 35 now [13]. However, the studies of grain segregation for 36 ripples under waves are scarce. A surface sorting is reported: 37 coarse sediments are observed to accumulate mostly along 38 the ripple crests, while fine grains mostly accumulate in the 39 troughs [14-16]. The presence of fine particles forming very no narrow strips on the top of crests is mentioned in [16]. In ${ }^{41}$ the case of standing waves, sand bar formation is observed 42 with bar crests composed of coarse sand and flat plateaus 43 of fine sand [17]. The density segregation under decaying 44 highly nonlinear waves, solitons, is considered in Ref. [18]. ${ }_{45}$ Light grains concentrate in a narrow region close to the ripple 46 crests when the waves are damped. The sorting process was 47 not analyzed in detail in Ref. [18]. The aim of the present 48 work is to study the segregation process above a rippled bed 49 under a simple oscillating flow, standing waves, from a detailed 50 analysis of the motion of individual particles of different sizes.

51 Grain trajectories under standing waves and decaying standing

52 waves are experimentally obtained, and concentrations of

53 sinking particles are measured before their deposition on the

\footnotetext{
‘tien-dat.chu@univ-lehavre.fr.
}

bed. There is only a restricted number of experimental studies on granular material dynamics dealing with trajectories of individual particles [19]. Present measurements improve the 50 theoretical model for the flow above ripple crests proposed in 57 Ref. [18]. Finally, a model for the flow above ripple troughs 58 is suggested to complete the explanation of the experimental 59 results.

This paper is organized as follows. In Sec. II, a short description of the experimental setup is given. The experimental results are presented in Sec. III. Sec. IV is devoted to the $f_{r}=0.31$ theoretical approach. The theoretical results are compared with the experimental findings. The paper ends with 65 conclusions in Sec. V.

\section{EXPERIMENTAL SETUP AND PROCEDURE}

The experiments were conducted in a 5.4-m-long, 0.3-m-wide wave flume. Surface waves are generated by an oscillating paddle at one end of the flume; a near-perfect 70 reflection takes place at the other end. The frequency of the 7 oscillating paddle is chosen close to the resonant frequency 72 $\mathrm{Hz}$ of the mode whose wavelength is equal to the effective 73 flume length $\left(L_{w}=5 \mathrm{~m}\right)$. The water depth at rest $h$ is $0.26 \mathrm{~m}$. The height and the period of the wave are measured with 75 two resistive probes. For the present tests, the maximum wave 76 height at the antinode $H$ is $0.04 \mathrm{~m}$. Ripples were generated 77 beneath standing waves from an initially flat bed, which 78 consisted in a 4-cm layer of polyvinyl chloride (PVC) grains 79 of density $\rho_{p}=1350 \mathrm{~kg} \cdot \mathrm{m}^{-3}$ and median diameter $d_{p}=80$ $0.20 \mathrm{~mm}$. Ripples formed rapidly along the flume once the 81 wave maker was switched on. Their size and shape change 82 from one end of the flume to the other end, depending on their 83 position in relation to the position of the nodes and antinodes 84 of the standing wave. The regions under the antinodes are 85 essentially flat, whereas the regions under the nodes where 86 the bed shear stress is maximum are covered by the greatest 87 ripples. When the equilibrium state is reached, we switch off the oscillating paddle and empty slowly the wave flume in 89 order to maintain the natural form of the bed. The rippled 90 bed is then covered by a thin powdering of cement. When 9 this powdering is dry, the wave flume is refilled with the same water level at rest as previously $(0.26 \mathrm{~m})$. PVC particles 93 are then carefully injected through the free surface above 


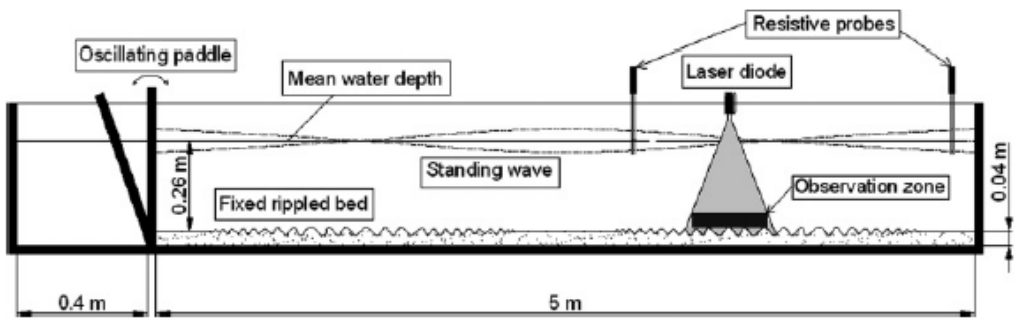

FIG. 1. Side view of the flume.

95 the fixed rippled bed, and their trajectories are registered 96 The flow conditions change along the wave flume and the ${ }_{97}$ Reynolds number, $\operatorname{Re}=U_{\infty} a / v$, varies in the range $0<\operatorname{Re}<$ ge 5700, where $U_{\infty}=a \omega=\pi H /[T \times \sinh (k h)]$ is the velocity 99 of fluid particles at the edge of the bed boundary layer, $a$ is 100 the orbital amplitude of fluid particles at the same place, $\omega$ 101 is the wave pulsation, $T$ is the wave period, $v$ is the water 102 kinematic viscosity, and $k$ is the wave number. The edge of 103 the bottom boundary layer may be estimated at the height $104 k_{s}$ above the level midway between crest and trough of the 105 ripples [20], where $k_{s}$ is the Nikuradse roughness length, 106 which may be estimated for a ripple bed by $k_{s}=25 H_{r}^{2} / L_{r}$

107 [21]. The studied zone for the particle trajectories and the 108 sediment sorting is chosen close to the node located in the 109 vicinity of the wall reflecting the surface waves (Fig. 1). 110 In this zone, ripples are mostly regular and bidimensional; 111 their wavelength $L_{r}$ and their height $H_{r}$ are, respectively, 5.5 112 and $1.5 \mathrm{~cm}$, and we have $\operatorname{Re} \approx 4500$. The mobility number ${ }_{113}(\Psi)$, the Stokes sedimentation velocity $\left(U_{0}\right)$, and the Stokes 114 number (St) are calculated as follows: $\Psi=(a \omega)^{2} / d_{p} g(s-1)$, $115 U_{0}=d_{p}^{2}\left(\rho_{p}-\rho_{w}\right) g / 18 v \rho_{w}$, and $\mathrm{St}=d_{p}^{2} \rho_{p} \omega / 18 v \rho_{w}$, where $116 \mathrm{~g}$ is the acceleration due to gravity, $s$ is the relative density 117 of grain, and $\rho_{w}$ is the fluid density. The Shields number $\theta$ 118 may be estimated with $\theta=0.5 f_{w} \Psi$, where $f_{w}$ is the friction 119 factor. Following Davies [22], the flow regime depends on the 120 values of $\operatorname{Re}$ and $a / k_{s}$; this regime is rough turbulent in the 121 considered zone. Five different kinds of PVC particles have 122 been used, as specified in Table I showing the experimental 123 conditions; the hydrodynamic forcing is the same for all of 124 the tests. Tests 1 to 5 were carried out with well size-sorted 125 particles $\left(P_{1}\right.$ to $\left.P_{5}\right)$, while Test 6 involved a mixture of $50 \%$ 126 (in weight) very fine particles $\left(P_{1}\right)$ and $50 \%$ medium particles ${ }_{127}\left(P_{3}\right)$ and Test 7 involved a mixture of $50 \%$ very fine particles ${ }_{128}\left(P_{1}\right)$ and $50 \%$ coarse particles $\left(P_{5}\right)$.

Grain trajectories were obtained performing particle track${ }_{130}$ ing velocimetry (PTV). The principle of PTV is to individually 131 follow each introduced particle in successive images. A 132 high-resolution camera Dalsa Falcon PT-41-4M60 (resolution $1332352 \times 1728$ pixels), 62 frames per second, is used in combi134 nation with a laser diode, which generates a thick vertical light 135 sheet. The thickness of the light sheet is fixed to two centime136 ters so that particles stay long enough in the illuminated area to ${ }_{137}$ enable the capture of grain trajectories over several wave peri138 ods $[23,24]$. Particle trajectories are obtained using a program 139 presented in Ref. [25] and adapted to the present experiments.

140 For present tests, two phases of the flow were considered: 141 during the excitation of standing waves [phase (1)] and during the decay of surface waves [phase (2)], after the stopping of the ${ }_{142}$ oscillating paddle. The dimensions of the observation window of the video camera are fixed to one ripple wavelength in ${ }_{144}$ the vertical direction, one ripple wavelength in the horizontal 145 direction for the phase 1 and four ripple wavelengths for ${ }_{140}$ the phase 2. The large horizontal area for phase 2 allows ${ }_{147}$ accurate study of the sediment sorting and the settling zones ${ }_{148}$ for decaying waves, when the horizontal zoom in on one ripple ${ }_{149}$ length for phase 1 provides very precise information on particle 150 trajectories close to the ripple crests, as shown in the next ${ }_{151}$ section.

\section{EXPERIMENTAL RESULTS}

A. Particle motion in the vicinity of ripple crests during the excitation of standing waves

Organized vortices are formed on the lee side of ripples and 156 shed above ripple crests each half-cycle [20,26]. Figure 2(a) depicts a typical trajectory above the rippled bed under the ${ }_{150}$ effect of vortices showing the complex motion of particles. 159 Let us focus on the area close to ripple crests. The oscillating 160 movement of particles in a zone confined just above the top of the ripple crests can be observed, as shown in Fig. 2(b). In this ${ }_{162}$ confinement zone, particles can be "captured" [Figs. 2(b) and 163 2(c)] and ejected in the flow several periods later [Fig. 2(d)]. Form and dimensions of the confined zone are determined by the extreme positions of grains that oscillate in it. Its lower 160 limit is the ripple crest and its height varies from zero at its ${ }_{167}$

TABLE I. Experimental conditions

\begin{tabular}{|c|c|c|c|c|c|c|}
\hline Particle & $s$ & $d_{p}(\mathrm{~mm})$ & $U_{0}(\mathrm{~mm} / \mathrm{s})$ & $U_{\infty} / U_{0}$ & St & $\Psi$ \\
\hline $\mathrm{P}_{1}$ & 1.35 & 0.11 & 2.1 & 51.4 & $1.6 \times 10^{-3}$ & 29.9 \\
\hline $\mathrm{P}_{2}$ & 1.35 & 0.19 & 5.8 & 18.5 & $4.5 \times 10^{-3}$ & 17.9 \\
\hline $\mathrm{P}_{3}$ & 1.35 & 0.28 & 13.2 & 8.2 & $10.2 \times 10^{-3}$ & 11.9 \\
\hline $\mathrm{P}_{4}$ & 1.35 & 0.45 & 33.4 & 3.22 & $25.8 \times 10^{-3}$ & 7.5 \\
\hline $\mathrm{P}_{5}$ & 1.12 & 0.57 & 18.1 & 5.93 & $33.7 \times 10^{-3}$ & 17.3 \\
\hline \multicolumn{3}{|c|}{ Test number } & \multicolumn{3}{|c|}{ Particle } & \\
\hline \multicolumn{3}{|l|}{1} & \multicolumn{3}{|c|}{$P_{1}$} & \\
\hline 2 & & & & & & \\
\hline 3 & \multicolumn{6}{|c|}{$P_{3}$} \\
\hline 4 & \multicolumn{6}{|c|}{$P_{4}$} \\
\hline 5 & \multicolumn{6}{|c|}{$P_{5}$} \\
\hline 6 & \multicolumn{6}{|c|}{$50 \% P_{1}-50 \% P_{3}$} \\
\hline 7 & \multicolumn{6}{|c|}{$50 \% P_{1}-50 \% P_{5}$} \\
\hline
\end{tabular}




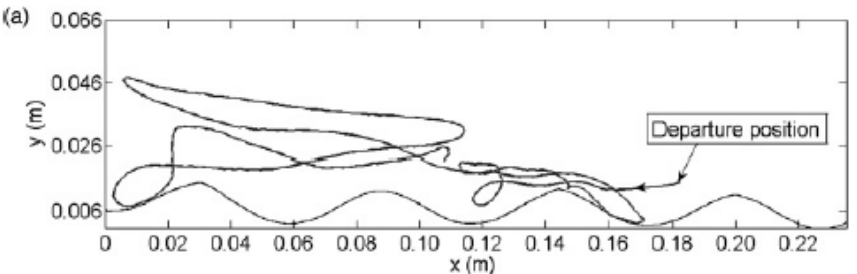

(b)
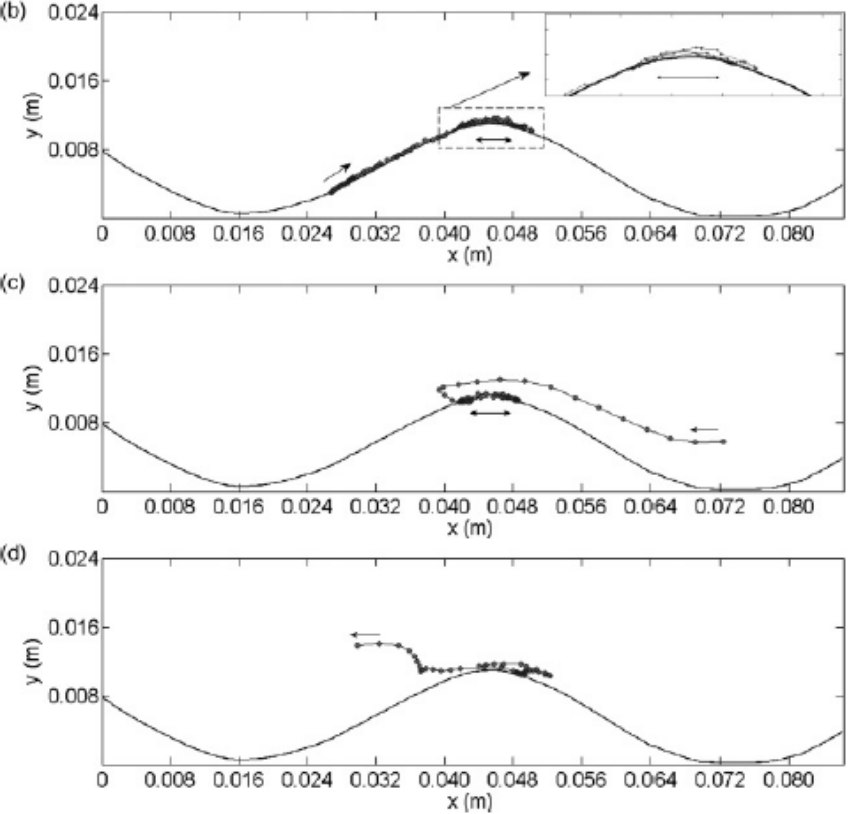

FIG. 2. Typical particle trajectories. (a) Particle trajectory under the action of vortices, Test 1; (b) Rolling of a particle along ripple side and "capture" in the confinement zone, Test 2; (c) "Capture" of a suspended particle, Test 2; (d) "Ejection" of a particle from the confinement zone, Test 4 .
168 extremities to $H_{c z}=0.9 \mathrm{~mm}$ at the top of the ripple crest; 169 this height is comparable with the Stokes layer thickness, $\delta=$ $170 \sqrt{2 v / \omega} \approx 1 \mathrm{~mm}$, and is greater than the grain size. The length $171 L_{c z}$ of the confinement zone may be estimated at $10.9 \mathrm{~mm}$, 172 which corresponds approximately to one-fifth of the ripple 173 wavelength in the tested zone. Present tests show that the 174 ejected particles are the ones for which the diameter $d_{p}$ is 175 greater than approximately $0.28 \mathrm{~mm}$. These particles are more 176 exposed to the vortices than finer ones. We measured the mean 177 resident time of particles in the confinement zone according 178 to the sediment size. This time varies from one-half to two 179 or three times the wave period for coarse particles. It is much 180 longer for very fine and fine particles, ranging from three times 181 the wave period to the total duration of the experiment. The 192 grains capture and ejection at ripple crests may be considered 193 as a sediment sorting process. The existence of a confinement 194 zone is taken into account in the theoretical approach which is 195 presented in Sec. IV.

B. Particle trajectories during the decay of surface waves

7 When the oscillating paddle is stopped, suspended particles 198 start to settle under the action of gravity. Let us consider that 199 the time $t=0$ is the instant corresponding to the stopping of the oscillating paddle. The settling velocity of the coarse 190 particles $\left(d_{p}=0.45 \mathrm{~mm}\right.$, Test 4$)$ is about 15 times larger ${ }_{191}$ than the one for the very fine particles $\left(d_{p}=0.11 \mathrm{~mm}\right.$, Test ${ }_{192}$ 1). As shown in Fig. 3(a), the amplitude of motion of coarse 193 particles before they reach the bed is large, since these particles 194 begin to settle rapidly after a few wave periods $(t \approx 2-3 \quad 195$ $T$ ) when the hydrodynamic forcing is still intense. Coarse 190 particles generally reach the bed after a trajectory composed of ${ }_{197}$ four lateral excursions in the observation window. On the other 198 hand, finer particles, which are lighter [Figs. 3(b)-3(d)], reach 199 the bed later when the amplitude of fluid motion is weaker; 200 the number of lateral excursions increases when particle size 201 decreases. The maximum number of lateral excursions is equal 202 to 10 for very fine particles $\left(d_{p}=0.11 \mathrm{~mm}\right)$. The ratio of the 203 amplitude of the lateral extension of particle excursion $a_{p}$ on 204 the amplitude of the fluid motion $a$ outside the boundary layer 205 in the dynamical phase [phase (1)] used as a reference fluid 200 amplitude is estimated. Typical values of $a_{p} / a$ are 0.45 and 0.1207 for coarse (Test 4) and very fine particles (Test 1), respectively, 200 when they arrive in the observation window. These values 209 decrease as particles approach the bed. About 100 trajectories 210 of sinking particles have been measured. Initial positions of 211 these particles at the upper limit of the observation window are 212 

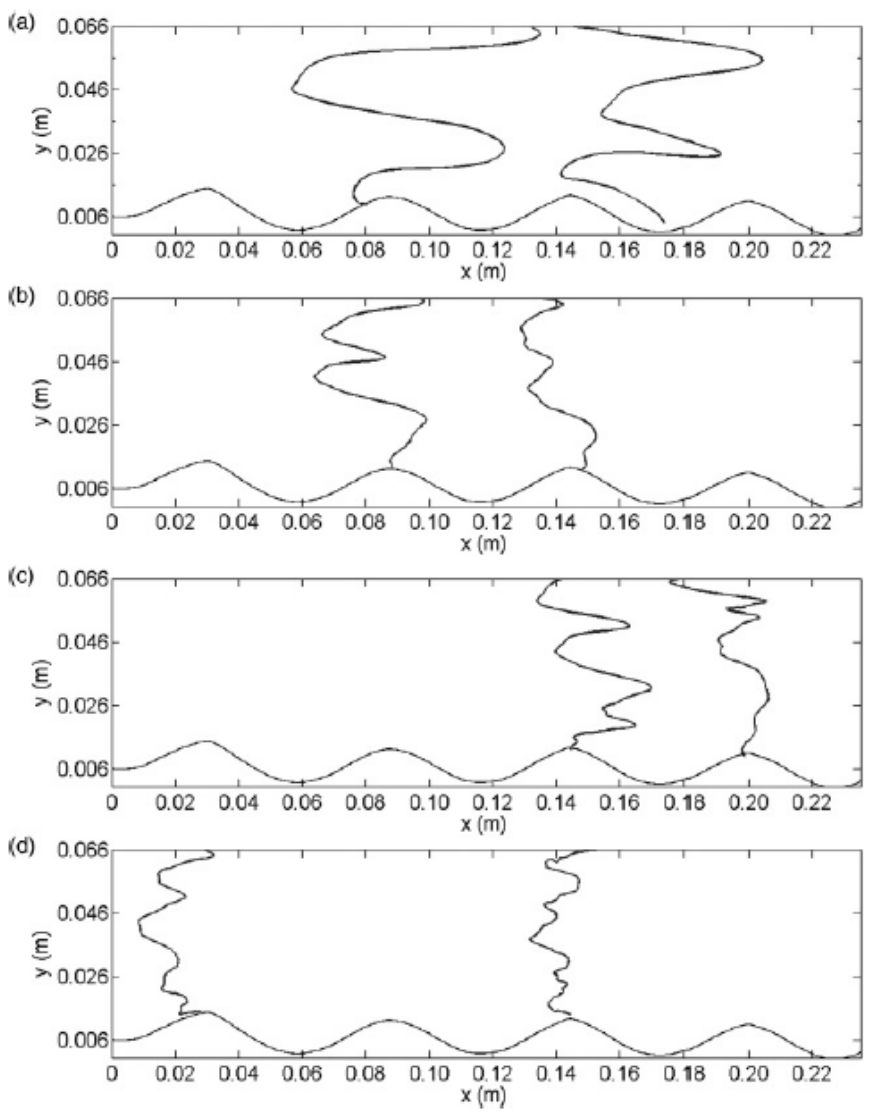

FIG. 3. Typical particle trajectories during the waves damping. (a) Test 4; (b) Test 3; (c) Test 2; (d) Test 1.
213 uniformly distributed above ripples. Places where the grains 214 settle along the rippled bed are analyzed according to their 215 size. The histogram of repartition of particles deposition zone 216 over the three regions delimiting the crest, trough, and both 217 sides is shown in Fig. 4. The length of the region defining the 218 vicinity of ripple crest is fixed to $10 \mathrm{~mm}$ (18\% of the ripple 219 wavelength in the considered zone) divided equally on both 220 sides of the top of the ripple. The length of the trough region 221 is estimated at $16 \mathrm{~mm}$; both sides occupy $29 \mathrm{~mm}$. The results 222 show that $61 \%$ of the very fine and medium particles (Tests 1 223 to 3 ) settle in the vicinity of the ripple crests, whereas $39 \%$ of 224 these particles settle elsewhere. In the case of coarse particles 225 (Tests 4 and 5), only $29 \%$ of grains settle in the vicinity 226 of the crests. Furthermore, for the range of coarse particles 227 considered herein, no privileged deposition place is noted. Let 228 us consider the temporal evolution of particle concentrations 229 just above the rippled bed when the surface waves damp.

\section{C. Suspension sorting during the decay of surface waves}

231 Figure 5 depicts the area in which particle concentrations 232 have been obtained; this area is a 1-cm-high horizontal strip. 233 Its lowest level is $2.9 \mathrm{~mm}$ above the highest point of the bed. 234 Zones delimiting regions above ripple crests ( $10 \mathrm{~mm}$ long) and above ripple troughs ( $16 \mathrm{~mm}$ long) are defined in the legend of 235 Fig. 5. Particle concentrations per unit area have been obtained 236 in the following way: the number of particles crossing the strip 237 is counted during their settling when the water waves damp. 239

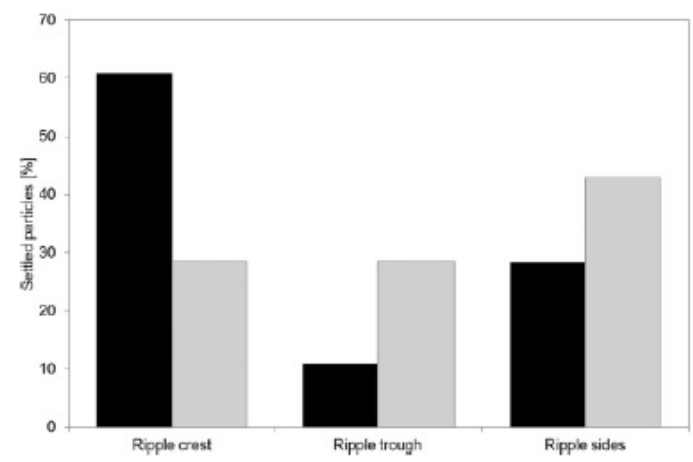

FIG. 4. Distribution of settled particles along the rippled bed surface. Black column: medium, fine, and very fine particles (Tests 1 , 2 , and 3, respectively); gray column: coarse particles (Tests 4 and 5). 


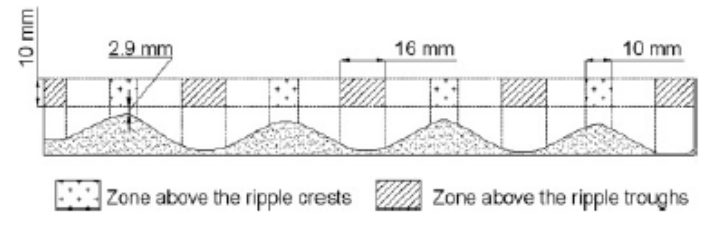

FIG. 5. Area above the rippled bed where particle concentrations have been measured.

239 The strip is in a vertical plane illuminated by a laser diode 240 (Fig. 1). The particles concentration $C$ is estimated along the 241 strip as $C=n / S$, where $n$ is the number of detected particles 242 in the considered zone and $S$ is the surface of this zone.

${ }_{243}$ The temporal evolution of relative concentration $C / C_{\max }$ is ${ }_{244}$ shown in Fig. 6, where $C_{\max }$ is the maximum concentration 245 measured over all the experiments during the settling process.

246 The relative error of concentration measurement is related to

${ }_{247}$ the particle detection process in the horizontal strip performed 248 in the Matlab program. It is estimated at $5 \%$ and depicted ${ }_{249}$ in Fig. 6 with error bars. The origin of temporal axis $t=0$ 250 corresponds to the time where the oscillating paddle is stopped. 251 This figure exhibits an exponential decrease of $C / C_{\max }$ with $252 t / T$. Using a best-fit procedure, the temporal variation of 253 particle concentration may be estimated with the following 254 equations:

$$
\begin{aligned}
C / C_{\max } & =1.49 e^{(-0.268 t / T)}, \\
0.45 & \leqslant d_{p} \leqslant 0.57 \mathrm{~mm}, \text { ripple crest and trough, } \\
C / C_{\max } & =1.16 e^{(-0.032 t / T)}, \\
0.11 & \leqslant d_{p} \leqslant 0.28 \mathrm{~mm}, \text { ripple crest, } \\
C / C_{\max } & =1.11 e^{(-0.039 t / T)}, \\
0.11 & \leqslant d_{p} \leqslant 0.28 \mathrm{~mm}, \text { ripple trough. }
\end{aligned}
$$

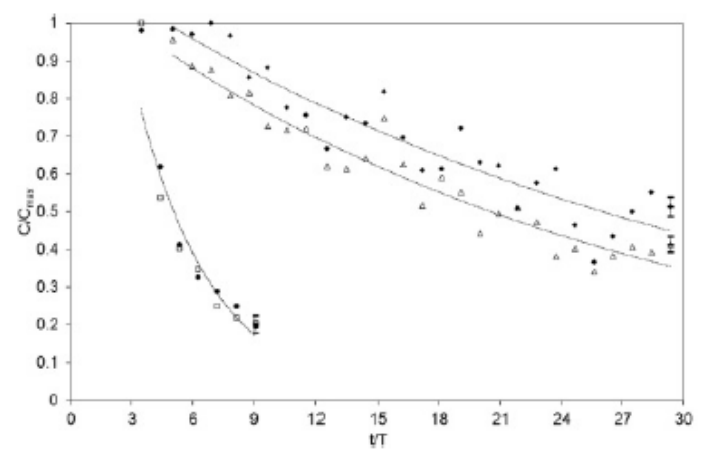

FIG. 6. Temporal evolution of particle concentrations close to the bed when the surface waves damp. Above ripple crest: $0.11 \leqslant d_{p} \leqslant 0.28 \mathrm{~mm}$ (Tests 1,2 , and 3 ). $\Delta$ Above ripple trough: $0.11 \leqslant d_{p} \leqslant 0.28 \mathrm{~mm}$ (Tests 1,2 ,and 3 ). $\bullet$ Above ripple crest: $0.45 \leqslant$ $d_{p} \leqslant 0.57 \mathrm{~mm}$ (Tests 4 and 5 ). $\square$ Above ripple trough: $0.45 \leqslant d_{p} \leqslant$ $0.57 \mathrm{~mm}$ (Tests 4 and 5 ). The curves represent the trendlines obtained using a best-fit procedure.
The particles in the size range $0.45 \leqslant d_{p} \leqslant 0.57 \mathrm{~mm}$ settle 255 rapidly and regularly without significant difference between 250 the concentrations above the ripple crests and troughs. As far 257 as the particles in the size range $0.11 \leqslant d_{p} \leqslant 0.28 \mathrm{~mm}$ are 258 concerned, the two plotted curves exhibit similar variations 259 and a trend to higher concentration above the crests that 200 can be interpreted as a size sorting above the rippled bed 261 during the particle settling when the surface waves damp. The 262 concentration distributions show temporal fluctuations in the 263 size range $0.11 \leqslant d_{p} \leqslant 0.28 \mathrm{~mm}$ when these distributions are 264 more regular for coarser grains. These are the result of the high 265 sensitivity of the light particles to the fluid movement until the 260 water becomes totally still.

D. Particle sorting after the decay of surface waves 268

In this section, we consider the final state for Tests 6 and 7, 269 when the water waves are damped. Figure 7(a) shows the grain 270 sorting at the end of Test 6 involving a mixture with $50 \%$ of ${ }_{271}$ very fine particles $\left(d_{p}=0.11 \mathrm{~mm}\right)$ and $50 \%$ of medium par- 272 ticles $\left(d_{p}=0.28 \mathrm{~mm}\right)$. Based on the observation of Fig. 7(a), 273 one ripple wavelength is split in five regions defined on this 274 figure: (i) designates the region on the top of the crest; (ii) is 275 the region nearby (i) directed toward the fixed end of the wave 278 flume; (iii), (iv), and (v) are, respectively, the ripple side toward 277 the fixed end of the flume (right ripple side), the ripple trough, 278 and the ripple side toward the oscillating paddle (left ripple 279 side). A calculation to estimate the proportions of covering for 290 each type of grain has been carried out. This process consists 281 in affecting ranges of color levels to very fine and medium 282 particles and in counting the number of pixels associated to the 283 two categories of grains in each region. The pixels uncovered 294 by particles are also counted. The results are reported in 285 Fig. 7(b) as percentages. Average values are calculated in each 290 zone. The top of the crest (i) is almost saturated in very fine ${ }^{287}$ particles covering $82 \%$ of this region, while the nearby region 288 (ii) is covered essentially by medium grains $(70 \%)$. For the 289 three other regions, very fine and medium particles are present 290 composing a mixture. For standing waves above a flat bed, 291 the steady drift takes the form of closed recirculating cells 292 with boundaries at a spacing of one-quarter the wavelength of 293 the surface waves $[27,28]$. Very close to the bed that is inside 294 the Stokes boundary layer thickness, the fluid particle drift 295 is oriented toward the node of the water surface profile and 296 away from the antinodes. However, the drift is in the opposite 297 direction further in the outer flow. Present tests are carried 298 out above a rippled bed, and vortices are generated leading 299 to suspended load transport. As shown in Fig. 1 (Sec. II), 300 the particle trajectories are recorded between the antinode of 301 the surface wave in the central part of the flume and the node 302 close to the fixed end of the channel, where the drift is oriented 303 toward the wave paddle, outside the Stokes layer. A possible 304 reason for the settling of medium particles on the right ripple 305 sides forming strips close to the ripple crests is the effect of drift ${ }_{300}$ velocity leading to vortices formed more vigorously during one 307 half-cycle than during the other. The vortices generated above 300 the left ripples sides are stronger than the ones formed above ${ }_{309}$ the right sides; at flow reversal, these vortices move toward the 310 right sides carrying particles. The medium particles settle on 311 the bottom when the vortices intensity is still high. The very 312 
(a)

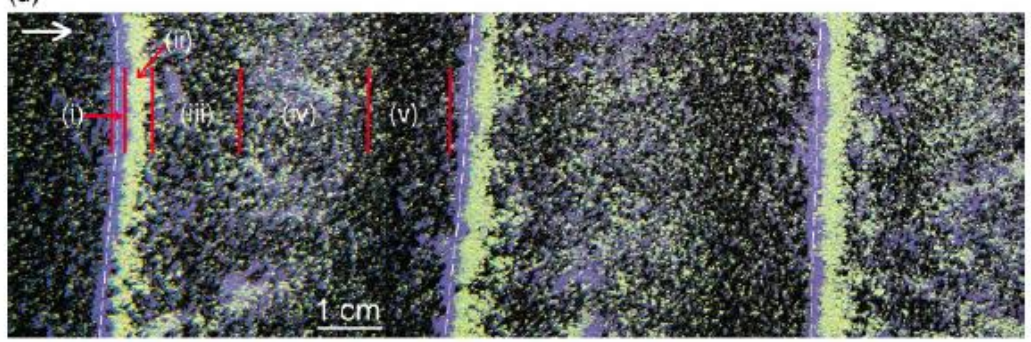

(b)

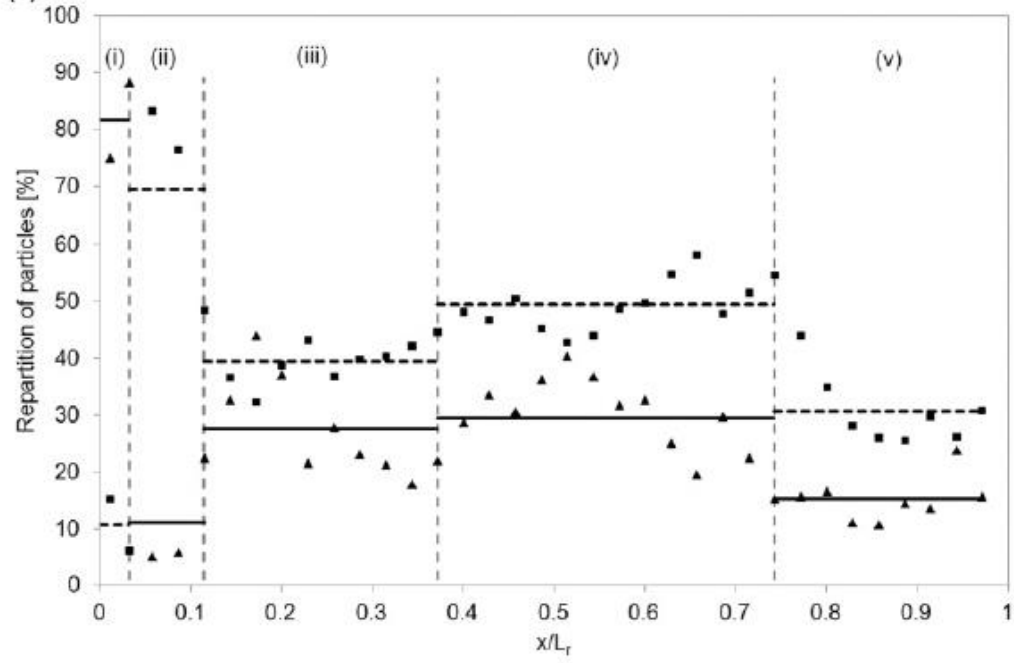

FIG. 7. (Color online) (a) Sediment sorting on the bed at the end of Test 6 . The dashed lines represent the ripple crests positions. The arrow depicts the direction from the wave paddle to the fixed vertical end of the flume. Ripple crests are covered by very fine particles [violet (gray) grains]; a strip of medium particles [light beige (white) grains] is located nearby each crest. Very fine and medium particles are observed on the ripple troughs and sides, (i)-(v) Designate the five regions along one ripple wavelength used to evaluate the particle covering. (b) Repartition of particles over one ripple wavelength at the end of Test 6. Limits between different regions (vertical dashed lines); averaged values of medium particles distribution (horizontal dashed lines); averaged values of fine particles distribution (horizontal solid lines). (4) Very fine particles; (ם) medium particles.

${ }_{313}$ fine particles fall later, when the vortices intensity is weaker 314 and the asymmetry becomes negligible.

For Test 7 , which was carried out with a mixture with 50 $316 \%$ of very fine particles $\left(d_{p}=0.11 \mathrm{~mm}\right)$ and $50 \%$ of coarse 317 particles $\left(d_{p}=0.57 \mathrm{~mm}\right)$, approximately the same particle 318 segregation was qualitatively observed as for Test 6 , the coarse 319 particles taking the role of the medium particles used for Test 3206 . We can just note that the relative narrow strips of medium 321 particles, which were observed in region (ii) for Test 6, were 322 wider for the coarse particles of Test 7 . These observations are 323 explained in the next section with a theoretical approach.

\section{THEORETICAL APPROACH}

325 We present in this section an improvement of the theoretica 326 model proposed in Ref. [18] for the flow above ripple crests and 327 a simple model for the flow above ripple troughs, to explain the present experimental results on the sorting of particles 29 under damping waves. The model presented in Ref. [18] $x_{3}$ was not based on a detailed analysis of particle trajectories 390 above a rippled bed; the process of grain segregation was 391 not considered in detail. Present experimental results for 332 particle trajectories show us in particular that close to ripple 303 crests, particles oscillate. The model previously developed in 396 Ref. [18], which had as specificity a fixed hyperbolic point 305 at ripple crest, had to be improved to take into account the 396 oscillating movement of particles in a small zone just above 337 the ripple crest (confinement zone). This consideration affects 398 the particle trajectories and the settling zones.

A. Improvement of the theoretical model proposed in Ref. [18] 340 for the flow above ripple crests

A very simple model in a narrow region near ripple crests 342 was considered in Ref. [18], taking into account only the Stokes 343 
(a)

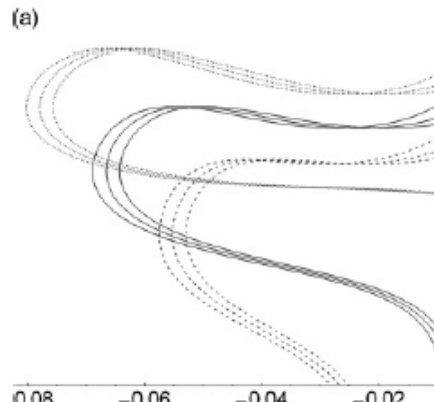

(b)

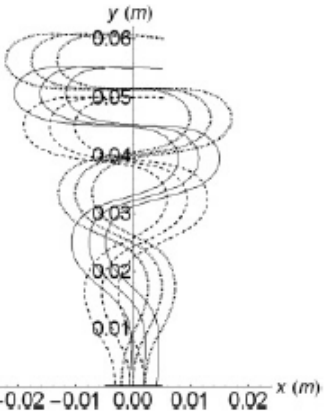

FIG. 8. Theoretical trajectories starting from nine different initial points above the ripple crest. (a) $d_{p}=0.45 \mathrm{~mm}$; (b) $d_{p}=0.28 \mathrm{~mm}$. The solid bold line on the $x$ axis depicts the area near the ripple crest.

344 force, and assuming in the first approximation that the bottom 345 is flat. The flow was modeled in the vicinity of each crest 346 by the stream function, $\psi=-a(\alpha x+y) y$, where $x$ and $y$ 347 are the horizontal and vertical coordinates. The point $x=$ 348 $0, y=0$ corresponds to ripple crests. The coefficient $\alpha=$ 349 $\alpha_{0} \sin (\omega t)$ is the parameter that controls the slope of the line 350 separating the forward and return flows. The value of $\alpha_{0}$ may 351 be estimated from present tests from flow visualization: $\alpha_{0} \approx$ $3521 \mathrm{rad}$. The coefficient $a=a_{0} \sin (\omega t)$ is related to the vorticity 35з $\vec{\Omega}=2 a \vec{z}$, where $\vec{z}$ is a unit vector perpendicular to the flow 354 plane. A slip condition is assumed on the bed surface $y=0$, 355 and the influence of viscosity was neglected outside the Stokes 356 layer. It was supposed that this thickness is much less than 357 the other characteristic scales of motion in the vicinity of the 358 ripple crest. A particularity of the flow was the presence of 359 a hyperbolic point with a stationary position at $x=0, y=0$. 360 Present experimental results show (Sec. III A) that close to 361 ripple crests, particles oscillate in a confinement zone. An 362 improvement of the model presented in Ref. [18] consists in 363 introducing an oscillating movement of the hyperbolic point. 364 We suggest modeling the flow in the vicinity of ripple crests ${ }_{365}$ by the following stream function: $\psi=-a\left[\alpha\left(x+x_{0}\right) y+y^{2}\right]$, 366 where $x_{0}=X_{0} \sin (\omega t), X_{0}$ is the amplitude of the movement 367 of hyperbolic point and is considered to correspond to half 368 of the length of the confinement zone. The horizontal $\left(U_{x}\right)$ 369 and vertical $\left(U_{y}\right)$ components of the flow velocity vector are з70 obtained from the stream function:

$$
\begin{gathered}
U_{x}=\frac{\partial \psi}{\partial y}=-a\left[\alpha\left(x+x_{0}\right)+2 y\right], \\
U_{y}=-\frac{\partial \psi}{\partial x}=a \alpha y .
\end{gathered}
$$

371 The particle velocity $\vec{V}$ does not coincide with the flow 372 velocity $\vec{U}$. For our experimental conditions, the Stokes зтз number is a small parameter $\left(S t \sim 10^{-3}, 10^{-2}\right)$ that we use ${ }_{374}$ as an expansion parameter. The particle velocity is written as

$$
\vec{V}=\vec{V}^{(0)}+\mathrm{St}^{(1)}+\mathrm{St}^{2} \vec{V}^{(2)}+\cdots
$$

Neglecting all terms proportional to $\mathrm{St}^{2}$ in Eq. (6), the 376 particle velocity field may be written as follows [18]:

$$
V_{x}=U_{x}-\frac{\mathrm{St}}{\omega} \sigma \frac{d U_{x}}{d t},
$$

$$
V_{y}=U_{y}-U_{0}-\frac{\mathrm{St}}{\omega} \sigma \frac{d U_{y}}{d t},
$$

where $V_{x}$ and $V_{y}$ are the projections of the particle velocity $a n$ vector onto the horizontal and vertical directions, and $\sigma=1-$ zre $\rho_{w} / \rho_{p}$. After the substitution of Eqs. (4) and (5) into Eqs. (7) 379 and (8), we obtain the following expressions for the particle 300 velocities close to the ripple crests:

$$
\begin{aligned}
V_{x}= & -a\left[\alpha\left(x+x_{0}\right)+2 y\right]+\frac{\mathrm{St}}{\omega} \sigma\left[(\dot{a} \alpha+a \dot{\alpha})\left(x+x_{0}\right)+2 \dot{a} y\right. \\
& \left.-a^{2} \alpha^{2}\left(x+x_{0}\right)+a \alpha \dot{x}_{0}\right], \\
& V_{y}=a \alpha y-U_{0}-\frac{\mathrm{St}}{\omega} \sigma\left[(\dot{a} \alpha+a \dot{\alpha}) y+a^{2} \alpha^{2} y\right] .
\end{aligned}
$$

It is supposed that after the oscillating paddle is stopped, the turbulence decays much more rapidly than the waves. During the wave damping, it is reasonable to consider that the vorticity 394 and the length of the confinement zone decay in the same way 325 as the amplitude of the surface waves. We suppose that the 396 change of the amplitude of particles velocity during a wave 387 period is small compared with the particles velocity amplitude. 398 The particles velocity temporally averaged over a wave period 399 $T=2 \pi / \omega$ is easily obtained from Eqs. (9) and (10):

$$
\begin{aligned}
& \left\langle V_{x}\right\rangle=-a_{0} \alpha_{0} e^{-2 \gamma t}\left[\frac{1}{2}+\frac{\mathrm{St}}{\omega} \sigma\left(\gamma+\frac{3}{8} a_{0} \alpha_{0} e^{-2 \gamma t}\right)\right] x, \\
& \left\langle V_{y}\right\rangle=a_{0} \alpha_{0} e^{-2 \gamma t}\left[\frac{1}{2}+\frac{\mathrm{St}}{\omega} \sigma\left(\gamma-\frac{3}{8} a_{0} \alpha_{0} e^{-2 \gamma t}\right)\right] y-U_{0},
\end{aligned}
$$

where $\gamma$ is the rate of exponential decay of surface waves.

The horizontal component $\left\langle V_{x}\right\rangle$ is negative for $x>0$ and positive for $x<0$, and particles tend to move toward 393 the ripple crest $(x=0)$ when the surface waves damp. The 394 rate of exponential decay for the present surface waves is 395 estimated at $\gamma=0.021 \mathrm{~s}^{-1}$ from measurements of the temporal 396 evolution of surface waves using resistive probes. Figure $8 \quad 397$ shows typical particle trajectories for different initial positions 399 and two median diameters: $d_{p}=0.45 \mathrm{~mm}$ and $d_{p}=0.28 \mathrm{~mm} . \quad 399$ The vertical coordinates of these initial positions are chosen 400 close to the upper bound of the observation window considered 401 in Secs. II and III (one ripple length high). The fine particles 402 

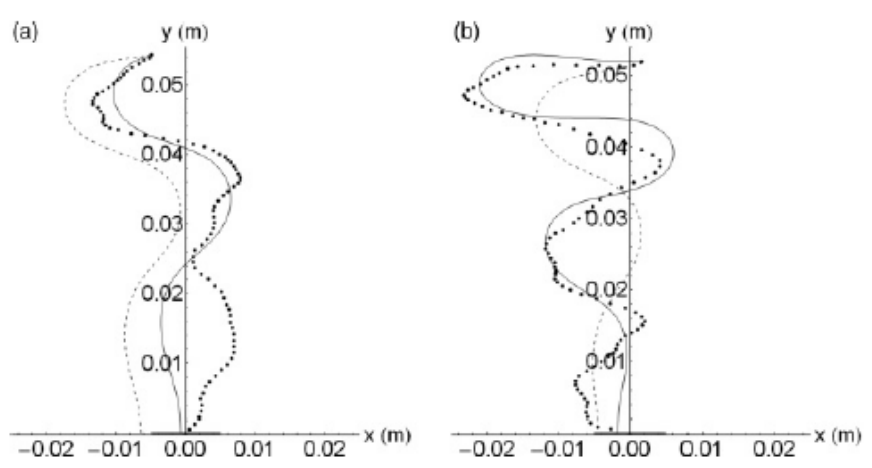

FIG. 9. Comparison of theoretical and experimental particle trajectories. (a) $d_{p}=$ $0.28 \mathrm{~mm}$; (b) $d_{p}=0.19 \mathrm{~mm}$. Present measurements (dotted line); Ezersky and Marin's (2008) model (dashed line); present model (solid line). The solid bold line on the $x$ axis depicts the area near the ripple crest.
${ }_{403}\left(0.11 \leqslant d_{p} \leqslant 0.28 \mathrm{~mm}\right)$ are found to settle close to ripple crests 404 when the coarser ones may fall everywhere on the bed. These ${ }_{406}$ particles settle on the bottom after only a few wave periods; ${ }_{406}$ Fig. 6 exhibits that most of them reach the area very close to 407 the bottom approximately for $4 \leqslant t / T \leqslant 9$, when the damping 408 time of surface waves is much greater $(1 / \gamma \approx 48 \mathrm{~s} \approx 15 T)$. ${ }_{409}$ For $d_{p} \leqslant 0.28 \mathrm{~mm}$, the time for particles to reach the bottom is 410 greater; Fig. 6 shows that when $t / T=30$, particles are not all 411 deposited on the bed. The settling area near the ripple crests is 412 well predicted by the present theoretical model. Experimental 413 and theoretical particle trajectories are depicted in Fig. 9; this 414 figure shows that the present adjustment of the model proposed 415 in Ref. [18] significantly improves the prediction of particle 416 trajectories and deposition areas.

417

\section{B. Model of flow above ripple troughs}

${ }_{418}$ The vorticity field above an artificial rippled bed under ${ }_{419}$ waves was considered in Ref. [20]. It was shown that the 420 values of the vorticity are much weaker above ripple troughs 421 than close to ripple crests (except very close to the bed). A 422 sketch of the rippled bed is depicted in Fig. 10. It can be seen 423 that the "angles of ripple troughs" slightly vary along the bed; 424 the averaged value is $135^{\circ}$.

425 Let us consider the classical model of potential flow for a ${ }_{426} 135^{\circ}$ angle:

$$
f(z)=\beta\left(z e^{-i \pi / 8}\right)^{4 / 3},
$$

427 where $z$ is a complex variable, $z=x_{1}+i y_{1}, i^{2}=-1$, and ${ }_{428} \beta=\beta_{a} \cos (\omega t)$ for an oscillating flow; the point $x_{1}=0, y_{1}=$ ${ }_{429} 0$ corresponds to ripple troughs. Using this model, which takes ${ }_{430}$ into consideration the curvature of the streamlines in the ripple 431 troughs, we are able to understand qualitatively why particles

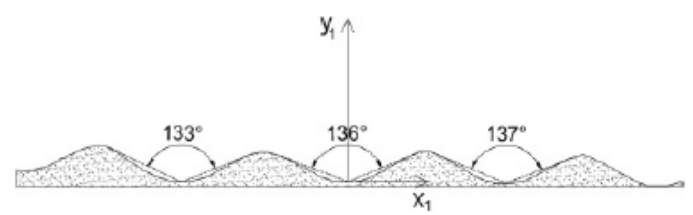

FIG. 10. Sketch of the rippled bed. settle in the ripple troughs. The complex function of the flow may be written in the following way:

$$
\begin{aligned}
f(z) & =\varphi+i \psi=\beta\left(r e^{i \theta} e^{-\frac{i \pi}{8}}\right)^{\frac{4}{3}} \\
& =\beta r^{\frac{4}{3}}\left(\cos \frac{4}{3}\left(\theta-\frac{\pi}{8}\right)+i \sin \frac{4}{3}\left(\theta-\frac{\pi}{8}\right)\right),
\end{aligned}
$$

where $\varphi$ and $\Psi$ are the potential and stream functions, 434 respectively, $r=\sqrt{x_{1}^{2}+y_{1}^{2}}$, and $\theta$ is the polar angle. Using 435 the Cartesian coordinates $U_{x_{1}}$ and $U_{y_{1}}$ for the flow velocity 436 components, we get

$$
\begin{gathered}
U_{x 1}=U_{r} \cos \theta-U_{\theta} \sin \theta=\frac{4}{3} \beta r^{1 / 3} \cos \left(\frac{\theta}{3}-\frac{\pi}{6}\right), \\
U_{y 1}=U_{r} \sin \theta+U_{\theta} \cos \theta=-\frac{4}{3} \beta r^{1 / 3} \sin \left(\frac{\theta}{3}-\frac{\pi}{6}\right),
\end{gathered}
$$

where $U_{r}$ and $U_{\theta}$ are the radial and orthoradial coordinates of 438 the flow velocity vector, respectively.

Substituting Eqs. (15) and (16) into Eqs. (7) and (8), we 40 get the instantaneous particle velocities after some transfor- 44 mations:

$$
\begin{aligned}
V_{x_{1}}= & \frac{4}{3} \beta\left(x_{1}^{2}+y_{1}^{2}\right)^{1 / 6} \cos \left[\frac{\arctan \left(y_{1} / x_{1}\right)}{3}-\frac{\pi}{6}\right] \\
& -\frac{\mathrm{St}}{\omega} \sigma\left\{\frac{4}{3} \dot{\beta}\left(x_{1}^{2}+y_{1}^{2}\right)^{1 / 6} \cos \left[\frac{\arctan \left(y_{1} / x_{1}\right)}{3}-\frac{\pi}{6}\right]\right. \\
& \left.+\frac{16}{27} \beta^{2}\left(x_{1}^{2}+y_{1}^{2}\right)^{-2 / 3} x_{1}\right\}, \\
V_{y_{1}}= & -\frac{4}{3} \beta\left(x_{1}^{2}+y_{1}^{2}\right)^{1 / 6} \sin \left[\frac{\arctan \left(y_{1} / x_{1}\right)}{3}-\frac{\pi}{6}\right]-U_{0} \\
& -\frac{\mathrm{St}}{\omega} \sigma\left\{-\frac{4}{3} \dot{\beta}\left(x_{1}^{2}+y_{1}^{2}\right)^{1 / 6} \sin \left[\frac{\arctan \left(y_{1} / x_{1}\right)}{3}-\frac{\pi}{6}\right]\right. \\
+ & \left.\frac{16}{27} \beta^{2}\left(x_{1}^{2}+y_{1}^{2}\right)^{-2 / 3} y_{1}\right\} .
\end{aligned}
$$


(a)

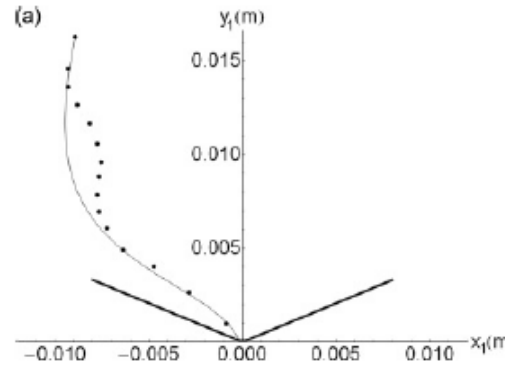

(b)

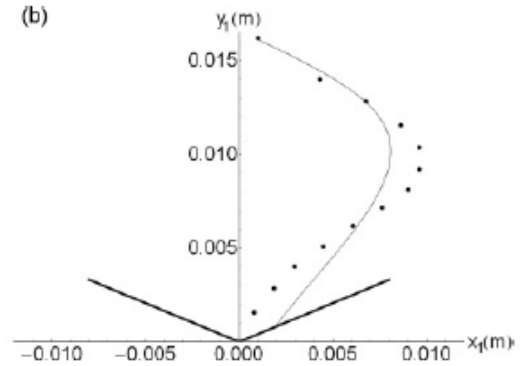

FIG. 11. Comparison between theoretical [Eqs. (17) and (18)] and experimental particle trajectories. Bold lines forming an angle represent the ripple trough region; present model (solid lines); present measurements (dotted lines). (a) $d_{p}=0.45 \mathrm{~mm}$; (b) $d_{p}=0.28 \mathrm{~mm}$.

450 theoretical trajectories are simpler than the experimental ones, 451 a qualitative agreement is obtained.

452 Assuming an exponential decay of the flow, $\beta=$ ${ }_{453} \beta_{a} \cos (\omega t) e^{-\gamma t}$, it is easy to obtain the particles velocity field 454 temporally averaged over one wave period:

$$
\begin{gathered}
\left\langle V_{x_{1}}\right\rangle=-\frac{8}{27} \frac{\mathrm{St}}{\omega} \sigma \beta_{a}^{2} e^{-2 \gamma t}\left(x_{1}^{2}+y_{1}^{2}\right)^{-2 / 3} x_{1}, \\
\left\langle V_{y_{1}}\right\rangle=-U_{0}-\frac{8}{27} \frac{\mathrm{St}}{\omega} \sigma \beta_{a}^{2} e^{-2 \gamma t}\left(x_{1}^{2}+y_{1}^{2}\right)^{-2 / 3} y_{1} .
\end{gathered}
$$

455 It should be noted that our model cannot be applied 456 for coordinates corresponding to positions very close to 457 the bed, because the influence of viscosity must be taken 458 into consideration for these positions. The averaged vertical 459 velocity of particles induced by the oscillating flow is directed 460 toward the ripple trough, due to centrifugal forces acting on 461 particles moving in a flow near a concave wall. The averaged 462 horizontal velocity of particles is negative for $x_{1}>0$ and 463 positive for $x_{1}<0$, and particles tend to converge toward 464 the ripple trough according to Eqs. (19) and (20). Using 405 Eqs. (11), (12), (19), and (20), it can be emphasized that 466 the ratio between the vertical and horizontal components of ${ }_{467}$ particle velocities is much smaller near the ripple crest than in 468 the ripple trough. It means that a particle in the vicinity of a 46 ripple crest will converge more strongly toward this crest than 470 a particle with the same diameter above a ripple trough will 471 converge toward the trough. Comparing Eq. (12) with Eq. (20) 472 for the averaged vertical velocity of particles, it is possible 473 to conclude that the particles settling time is greater near the 474 ripple crest than near the ripple trough. The centrifugal effect ${ }_{475}$ near the concave wall leads to an averaged velocity in the same 476 direction as the gravity force. In other words, coarse particles 477 settle rapidly on the bed before the damping of surface waves, 478 and fine particles with smaller settling velocities are more 479 affected by the mean horizontal velocities toward the ripple 400 crest than coarser particles. Then, an accumulation of fine 481 particles appears at ripple crests when the surface waves are completely damped, as observed in the present experiments 482 (Sec. III).

\section{CONCLUSIONS}

This paper presents an experimental and theoretical study 495 of particle size sorting above an artificial rippled bed under 496 standing water waves. Present tests were carried out in a 5.4- 487 $\mathrm{m}$-long wave flume with lightweight grains; two phases were 48 considered: during the excitation of standing waves [phase (1)] 499 and during the decay of surface waves [phase (2)]. Particle 490 trajectories were obtained for the two phases with a high- 491 resolution camera. In the first phase, fine and medium particles 492 $\left(0.11 \leqslant d_{p} \leqslant 0.28 \mathrm{~mm}\right)$ are trapped in a thin confinement zone, 493 approximately one Stokes boundary layer thickness high, close 494 to ripple crests. In the second phase, trajectories and deposition 495 zones of particles on the rippled bed strongly depend on their 496 size. Most of fine and medium particles fall in the vicinity of ${ }_{497}$ ripple crests when coarser particles $\left(0.45 \leqslant d_{p} \leqslant 0.57 \mathrm{~mm}\right) \quad 498$ settle more uniformly along the rippled bed, with nevertheless 499 an asymmetry in the deposition zones due to the mean flow 500 induced by standing waves near the bottom. Measurements 501 of particles concentration close to the bottom show a size 502 segregation during the wave damping, when particles are still 503 in suspension: the concentrations of the finest tested particles 504 is higher above the ripple crest than above ripple trough, when 505 no significant difference can be noted for coarser particles. 506 An improvement of the model proposed in Ref. [18] for the 507 flow above ripple crests and a simple model for the flow above 508 ripple troughs explain the present experimental results. Further 509 research is needed to consider particle size segregation with 510 active beds.

\section{ACKNOWLEDGMENT}

512

Financial support by the Haute-Normandie region ("Scale" ${ }_{513}$ Network) is gratefully acknowledged.
[1] H. Ayrton, Proc. R. Soc. London, Ser. A 84, 285 (1910).

[2] R. A. Bagnold, Proc. R. Soc. London, Ser. A 187, 1 (1946).

[3] T. Schnipper, K. Mertens, C. Ellegaard, and T. Bohr, Phys. Rev. E 78, 047301 (2008).
[4] K. H. Andersen, M. L. Chabanol, and M. V. Hecke, Phys. Rev. E 63, 066308 (2001).

[5] A. G. Davies and P. D. Thorne, J. Geophys. Res. 110, C05017 (2005). 
[6] V. Marieu, P. Bonneton, D. L. Foster, and F. Ardhuin, J. Geophys. Res. 113, C09007 (2008).

[7] T. Hara, C. C. Mei, and K. T. Shum, Phys. Fluids A 4, 1373 (1992).

[8] H. C. Earnshaw and C. A. Greated, Exp. Fluids 25, 265 (1998).

[9] T. S. Jespersen, J. Q. Thomassen, A. Andersen, and T. Bohr, Eur. Phys. J. B 38, 127 (2004).

[10] P. Nielsen, J. Geophys. Res. 86, 6467 (1981).

[11] G. Vittori and P. Blondeaux, J. Fluids Mech. 218, 19 (1990).

[12] J. Lebunetel-Levaslot, A. Jarno-Druaux, A. B. Ezersky, and F. Marin, Phys. Rev. E 82, 032301 (2010).

[13] I. S. Aranson and L. S. Tsimring, Rev. Mod. Phys. 78, 641 (2006).

[14] E. Foti and P. Blondeaux, Coastal Eng. 25, 237 (1995).

[15] J. S. Doucette, Sedimentology 49, 483 (2002).

[16] S. Balasubramanian, S. I. Voropayev, and H. J. S. Fernando, J. Turbulence 9, 1 (2008).

[17] B. J. Landry, M. J. Hancock, and C. C. Mei, Coastal Eng. 54, 694 (2007)
[18] A. B. Ezersky and F. Marin, Phys. Rev. E 78, 022301 (2008).

[19] J. M. Pastor, D. Maza, I. Zuriguel, A. Garcimartin, and J. F. Boudet, Physica D 232, 128 (2007).

[20] F. Marin, Coastal Eng. 50, 139 (2004).

[21] D. H. Swart, Delft Hydraulics Laboratory Report No. R968. 1976.

[22] A. G. Davies, Coastal Eng. 4, 23 (1980).

[23] F. Hering, C. Leue, D. Wierzimok, and B. Jăhne, Exp. Fluids 23, 472 (1997)

[24] F. Hering, C. Leue, D. Wierzimok, and B. Jăhne, Exp. Fluids 24, 10 (1998).

[25] S. S. Rogers, T. A. Waigh, X. Zhao, and J. R. Lu, Phys. Biol. 4, 220 (2007)

[26] J. J. Van der Werf, V. Magar, J. Malarkey, K. Guizien, and T. O'Donoghue, Cont. Shelf Res. 28, 1040 (2008).

[27] K. Gislason, J. Fredsoe, R. Deigaard, and B. M. Sumer, Coastal Eng. 56, 341 (2009).

[28] M. S. Longuet-Higgins, Philos. Trans. R. Soc. London, Ser. A 245, 535 (1953). 